\title{
TEMPLATES AND TRAIN TRACKS
}

\author{
GEORGE FRANK
}

\begin{abstract}
Within the context of Smale flows on compact manifolds, this article deals with a relationship between abstract templates, branched 1-manifolds (train tracks), and laminations representing unstable separatrices of basic sets. We show that an abstract template, the richest in information of the above three entities, determines a member of each of the remaining two groups, and partial determinations in other directions are developed. As a result of this relationship, an obstruction to the realization of certain abstract templates in nonsingular Smale flows on homology 3-spheres is raised.
\end{abstract}

1. Introduction. Smale flows on 3-manifolds have come under study recently, with results by Franks and Williams among many others. Franks' Nonsingular Smale flows on $S^{3}[\mathbf{6}]$ answered many questions and, unsurprisingly, prompted more. The major question answered was: What combinations of 1-dimensional basic sets can occur in Smale flows on $S^{3}$ ? The pertinent information is contained in a Lyapunov graph - a graph where vertices are labeled with chain-recurrent pieces of a flow on a compact space. In fact, the label of a suspension of a subshift of finite type in the above article is a nonnegative matrix. In the present paper, we begin an investigation into another symbol for these basic sets: A signed matrix (structure matrix) with certain order data included. Eventually, we shall try to determine which collections of these can be realized in $S^{3}$; so far, we just notice that in the above context, an order array $A$ (definition within) determines a unique abstract ordered branched 1-manifold $K$ : a smooth graph where the order of branches at each branch point matters. $K$ then determines an orientable surface of unique minimal genus $g$; that is, to embed $K$ in a surface $S$, the genus of $S$ must be $\geq g$. When a basic set $\Lambda$ corresponding to $A$ is embedded in a nonsingular flow on a compact oriented 3-manifold $M$ with $H_{1}(M ; Q)=0, K$ must be embedded in a surface $N$ which is transverse to the flow on $M$; because such $N$ must be tori, we have

THEOREM A. A necessary condition for $A$ to be realized in a nonsingular flow on a homology 3-sphere is that $g \leq 1$.

An example of a basic set whose genus (in the sense of the above paragraph) is greater than 1 appears after Theorem 4.3 .

As we approach the above issue, we notice that the branched 1-manifolds are fundamental to the following problems: (1) For 1-dimensional basic sets $\Lambda$ in flows on 3-manifolds, describe $W^{u}(\Lambda) \cap \partial X$, where $X$ is an isolating neighborhood of $\Lambda$, and (2) To what extent does an abstract ordered branched 1-manifold determine a

Received by the editors May 5, 1986 and, in revised form, May 11, 1987.

1980 Mathematics Subject Classification (1985 Revision). Primary 58F15, 58F25. 
1-dimensional basic set? We have

THEOREM B. Assume $\Lambda$ is a basic set of a Smale flow on a compact orientable 3-manifold $M$ corresponding to an order array $A$ with derived branched 1-manifold $K$, and $g$ is a Lyapunov function (defined within) for $M$ such that $g(\Lambda)=c$. If $S$ is a component of $g^{-1}(c-\varepsilon)$ for $\varepsilon$ sufficiently small, $W^{u}(\Lambda) \cap S$ is a finite number of circles together with sets of the form $C \times \mathbf{R}, C$ a Cantor set, where $C \times t$ approaches a circle asymptotically as $t \rightarrow \infty$ and likewise for $t \rightarrow-\infty$; furthermore, $K$ carries $W^{u}(\Lambda) \cap S$. An analogous result holds for $W^{s}(\Lambda)$.

We use a combing procedure discussed by Williams in [8] to introduce an equivalence relation on a set of branched 1-manifolds and produce

THEOREM C. An equivalence class of branched 1-manifolds with pairing and squeezing data (defined herein) determines a unique flow equivalence class of the associated order arrays, possibly the empty class.

The above results shed a bit more light on Smale flows in dimension 3; in particular, they aid in providing a naive schematic picture of the situation. By using templates to approximate 1-dimensional basic sets, the results of Theorem $\mathrm{B}$ are evident, and we imagine constructing Smale flows by a series of identifications, gluing template boundaries-branched 1-manifolds - to surfaces, using rules delineated in a Lyapunov graph.

The author would like to thank John Franks for germinating and guiding this work, and the referee for many helpful suggestions.

2. Background and definitions. We will assume throughout, unless explicitly stating otherwise, that $\phi_{t}$ is a smooth flow on a compact manifold $M$.

DEFINITION 2.1. If $\phi_{t}$ is a flow on $M$ and $x \in M$, we say that $x$ is chain-recurrent if for any $\varepsilon>0$ there exist points $x_{1}=x, x_{2} \ldots, x_{n}=x$ and real numbers $t(i) \geq 1$ such that $d\left(\phi_{t(i)}\left(x_{i}\right), x_{i+1}\right)<\varepsilon$ for all $1 \leq i<n$. The set of all such points, called the chain-recurrent set $R$, is a compact invariant set under the flow.

DEFINITION 2.2. If $\phi_{t}$ is a flow on $M$, then a smooth function $g: M \rightarrow \mathbf{R}$ is called a Lyapunov function provided (a) $d\left(g\left(\phi_{t}(x)\right)\right) / d t<0$ for $x \notin R$, the chainrecurrent set; (b) For $x, y \in R, g(x)=g(y)$ if and only if for each $\varepsilon>0$ there exist points $x_{1}=x, x_{2}, \ldots, x_{n}=y, x_{n+1}, \ldots, x_{2 n}=x$ in $R$ and real numbers $t(i)>1$, $1 \leq i<2 n$, such that $d\left(\phi_{t(i)}\left(x_{i}\right), x_{i+1}\right)<\varepsilon, 1 \leq i<2 n$.

Results of [2 and 10] imply that any smooth flow on a compact manifold possesses a Lyapunov function.

A compact invariant set $\Lambda$ of a flow $\phi_{t}$ on $M$ has a hyperbolic structure if there is a continuous splitting of the tangent bundle $T_{\Lambda} M=E^{s} \oplus E^{u} \oplus E^{c}$ preserved by $D \phi_{t}$ for all $t$, where $E^{c}$ is spanned by the vector field tangent to the flow, and there exist constants $C, a>0$ such that

$$
\begin{aligned}
& \left\|D \phi_{t}(v)\right\| \leq C e^{-a t}\|v\| \text { for } v \in E^{s}, t \geq 0, \\
& \left\|D \phi_{t}(v)\right\| \geq C^{-1} e^{a t}\|v\| \text { for } v \in E^{u}, t \geq 0 .
\end{aligned}
$$

THEOREM 2.3 [7]. If the chain-recurrent set $R$ of a flow $\phi_{t}$ on a compact manifold $M$ has a hyperbolic structure, $R$ is a finite disjoint union of compact 
invariant sets $\Lambda_{1}, \Lambda_{2}, \ldots, \Lambda_{n}$ and each $\Lambda_{i}$ has a point whose forward orbit is dense in $\Lambda_{i}$.

The $\Lambda_{i}$ are called basic sets for the flow; by Definition 2.2(b), any Lyapunov function is constant on each $\Lambda_{i}$.

PROPOSITION $2.4[6]$. Suppose that $\phi_{t}$ is a flow on a compact oriented manifold $M$ with Lyapunov function $g: M \rightarrow \mathbf{R}$ and that $R$ has finitely many chain-transitive pieces. If $\Gamma$ is the quotient space of $M$ obtained by identifying each component of a level set of $g$ to a point, then $\Gamma$ is a finite graph; furthermore, if $H_{1}(M ; Q)=0, \Gamma$ is a tree.

$\Gamma$ is called a Lyapunov graph if we label each vertex with its chain-transitive piece.

DEFINITION 2.5. For $x \in \Lambda$, a compact invariant hyperbolic set for $\phi_{t}$, define the stable and unstable manifold of $x$ :

$$
\begin{aligned}
& W^{s}(x)=\left\{y \in M \mid \text { for some } r \in \mathbf{R}, d\left(\phi_{t}(y), \phi_{t+r}(x)\right) \rightarrow 0 \text { as } t \rightarrow \infty\right\}, \\
& W^{u}(x)=\left\{y \in M \mid \text { for some } r \in \mathbf{R}, d\left(\phi_{t}(y), \phi_{t+r}(x)\right) \rightarrow 0 \text { as } t \rightarrow-\infty\right\} ;
\end{aligned}
$$

we say $W^{s}(\Lambda)=\bigcup_{x \in \Lambda} W^{s}(x)$ and $W^{u}(\Lambda)=\bigcup_{x \in \Lambda} W^{u}(x)$.

We say that a flow $\phi_{t}$ on $M$ with hyperbolic chain-recurrent set $R$ satisfies the strong transversality condition if for each $x, y \in R, W^{s}(x) \Phi W^{u}(y)$ (transverse intersection).

DEFINITION 2.6. A smooth flow $\phi_{t}$ on a compact manifold $M$ is called a Smale flow provided:

(1) its chain-recurrent set $R$ has a hyperbolic structure and $\operatorname{dim} R \leq 1$;

(2) it satisfies the strong transversality condition.

For Smale flows, Bowen has completely categorized the dynamics on each basic set, up to topological equivalence.

THEOREM $2.7[\mathbf{1}]$. Suppose $\phi_{t}$ is a flow with hyperbolic chain-recurrent set and $\Lambda$ is a 1-dimensional basic set. Then $\phi_{t} \mid \Lambda$ is topologically equivalent to the suspension of a subshift of finite type with an irreducible matrix.

We are then prompted to discuss suspensions of subshifts of finite type. Given an $n \times n$ nonnegative integer matrix $A$ of zeroes and ones, let

$$
\Sigma_{A}=\left\{\underline{x} \in \prod_{-\infty}^{\infty}\{1, \ldots, n\} \mid A_{x_{k} x_{k+1}}=1 \text { for all } k\right\}
$$

and define the right shift map $\sigma_{A}: \Sigma_{A} \rightarrow \Sigma_{A}$ by $\sigma_{A}(\underline{x})=\underline{y}$ where $y_{k}=x_{k-1}$. Then $\Sigma_{A}$ is a compact 0-dimensional space and $\sigma_{A}$ is called a subshift of finite type; it has topological transitivity if it has a dense orbit, and this is so if $A$ is irreducible: for each $i, j$ with $1 \leq i \leq n, 1 \leq j \leq n$ there is an $N>0$ such that $\left(A^{N}\right)_{i j} \neq 0$.

DEFINITION 2.8. If $h: X \rightarrow X$ is a homeomorphism then its suspension flow is defined on the identification space $Y=X \times \mathbf{R} /(x, s+1) \sim(h(x), s)$, and is defined to be the flow on $Y$ induced by the flow $\phi_{t}$ on $X \times \mathbf{R}$ given by $\phi_{t}(x, s)=(x, s+t)$. If $X=\Sigma_{A}$, we write $Y=X_{A}$. 
Different matrices can represent equivalent dynamics:

DEFInITION 2.9. Two nonnegative integer matrices $A$ and $B$ are said to be flow equivalent provided the suspended subshifts of finite type $X_{A}$ and $X_{B}$ are topologically equivalent.

A useful result is

THEOREM 2.10 [5]. Suppose that $A$ and $B$ are nonnegative irreducible integer matrices neither of which is a permutation matrix. Then $A$ and $B$ are flow equivalent if and only if: (1) $\operatorname{det}\left(I_{n}-A\right)=\operatorname{det}\left(I_{m}-B\right)$, and (2) $Z^{n} /\left(I_{n}-A\right) Z^{n} \cong$ $Z^{m} /\left(I_{m}-B\right) Z^{m}$, where $n$ and $m$ are the sizes of $A$ and $B$ respectively and $I_{n}$ and $I_{m}$ are identity matrices.

Suppose that $U \subset V$ are compact surfaces (with boundary) and that $f: U \rightarrow V$ is a diffeomorphism onto its image. Let $H=\bigcup_{i} h_{i}$ be a finite set of 1-handles in $U$-that is, each $h_{i}$ is an embedded copy of $D^{1} \times D^{1}$ where $D^{1}=[-1,1]$.

If $x$ is a point of $D^{1} \times p \subset h_{i}, p \in D^{1}$, we write $W_{i}^{u}(x)=D^{1} \times p$; similarly $W_{i}^{s}(x)$ will denote the interval $q \times D^{1}$ in $h_{i}$ containing $x$.

DEFINITION 2.11. The embedding $f: U \rightarrow V$ is hyperbolic with respect to the set of handles $H$ provided:

(1) if $x \in h_{i}$ and $f(x) \in h_{j}$, then $\operatorname{int}\left(f\left(W_{i}^{u}(x)\right)\right) \supset W_{j}^{u}(f(x))$ and $f\left(W_{i}^{s}(x)\right) \subset$ $\operatorname{int}\left(W_{j}^{s}(f(x))\right)$; and

(2) there is a $\lambda \in(0,1)$ such that for each $x \in h_{i}$ with $f(x) \in H$ and each $v \in$ $T_{x}\left(W_{i}^{s}(x)\right), w \in T_{x}\left(W_{i}^{u}(x)\right)$ we have $\|D f(v)\| \leq \lambda\|v\|$ and $\|D f(w)\| \geq \lambda^{-1}\|w\|$.

We then see that $\Lambda=\bigcap_{n \in Z} f^{n}(H)$ has a hyperbolic structure and that $W_{i}^{s}(x)$ and $W_{i}^{u}(x)$ are contained in the stable and unstable manifolds of $x$ respectively.

DEFINITION 2.12. The geometric intersection matrix $G$ associated to $f$ and $H$ is defined by $G_{i j}=$ number of components of $f\left(h_{j}\right) \cap h_{i}=$ number of points of intersection of $W_{i}^{s}(x)$ and $f\left(W_{j}^{u}(y)\right)$ for any $x \in h_{i}, y \in h_{j}$.

Then the suspension of $f \mid \Lambda$ is topologically equivalent to the suspension of the subshift of finite type $\sigma_{G}: \Sigma_{G} \rightarrow \Sigma_{G}$ (see [6]).

If $\Lambda_{i}$ is a basic set corresponding to a hyperbolic handle set $H$, we call $K\left(\Lambda_{i}\right)=$ $\bigcup_{t \in \mathbf{R}} \phi_{t}(H)$ the saturated handles.

We define a strip $k$ to be an embedded 1-handle; images of $D^{1} \times p$ are in the core foliation of $k$, and images of $q \times D^{1}$ in its transverse foliation. We call the images of $\{-1\} \times D^{1}$ and $\{1\} \times D^{1}$ the ends of $k$.

DEFINITION 2.13. A foliated train track neighborhood $N$ in a surface $V$ is a finite union of strips $\bigcup k_{i}$ such that

(1) if $k_{i} \cap k_{j} \neq \varnothing$, it consists of an end of $k_{i}$ either embedded in the interior of, or equal to, an end of $k_{j}$. Each end of a strip intersects some other strip;

(2) the foliations on the interiors of all the handles taken together extend to foliations on int $N$.

PROPOSITION $2.14[6]$. Let $\phi_{t}$ be a Smale flow on a 3-manifold $M$ with Lyapunov function $g$, and suppose $\Lambda$ is a basic set of $\phi_{t}$ associated to a handle set $H$ with $g(\Lambda)=c$. If $S=a$ component of $g^{-1}(c-\varepsilon)$ for $\varepsilon$ sufficiently small, and $S$ and $\Lambda$ are in the same component of $g^{-1}([c-\varepsilon, c+\varepsilon])$, then $N^{-}=S \cap K(\Lambda)$ is a foliated train track neighborhood. An analogous result holds for $P$, an appropriate component of $g^{-1}(c+\varepsilon)$. 
Williams' very useful idea of employing branched manifolds in this field plays an integral role here-we consider branched manifolds of dimensions 1 and 2: a branched 1-manifold or train track is a 1-dimensional subcomplex $K$ of a surface $F$ with the following properties.

(1) The branches (edges) are $C^{1}$ and are tangent at the vertices.

(2) At least 3 edges emanate from each vertex.

(3) Each vertex lies in the interior of a $C^{1}$ arc embedded in $K$.

By an abstract (ordered) branched 1-manifold, we will mean a 1-dimensional subcomplex $K$ of $S^{3}$ where a neighborhood (in $K$ ) of each vertex is smoothly embedded in $S^{2}$, along with properties 1-3 above. A fixed orientation of $S^{2}$ gives a cyclic order on the edges of $K$ at each branch point (vertex).

We shall consider only those branched 1-manifolds with a (locally) single-sheeted side: On at least one (of the two) side of each branch point $x$ there is only one branch connected to $x$. We shall occasionally refer to this single-sheeted side as a 0 -branch.

A lamination $L$ in a surface $F$ (a foliation of a closed subset of $F$ ) is carried by a train track $K$ if $L$ and $K$ are $C^{1}$-close.

We shall now discuss branched 2-manifolds, herein restricted to the concept of abstract templates. Consider a block $T_{i}$ as in Figure 2.1. We have drawn a partial flow on $T_{i}$ which is given by the unit downward vector field. The flow exits $T_{i}$ on the $\beta_{i}$ 's and the "arch intervals" between them, and is tangent to the vertical sides.

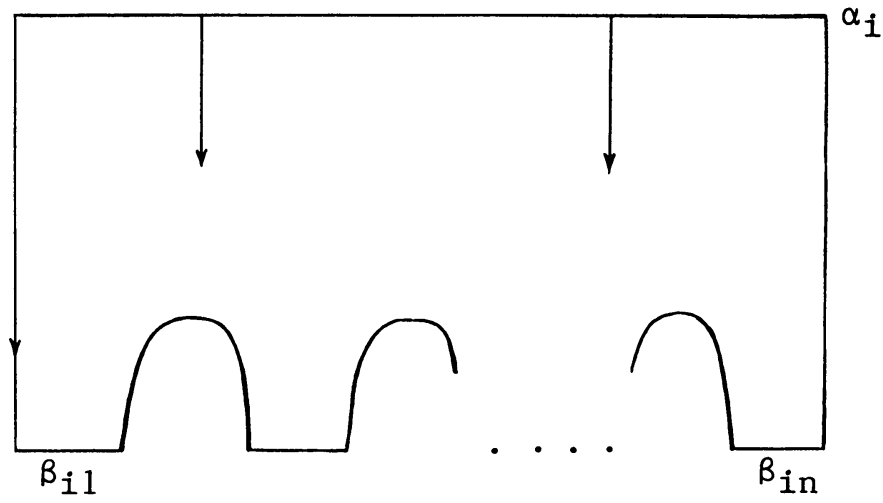

FIGURE 2.1

We form a branched surface $T$, with boundary, by gluing together a finite number of blocks $T_{i}$, attaching each $\beta_{i j}$ to an $\alpha_{k}$ and vice versa. We do this in such a way that each attaching map is overflowing and expanding; that is, we require $\alpha_{k}$ to be in the interior of $\beta_{i j}$ and that the attaching map from any interior subinterval of $\beta_{i j}$ to $\alpha_{k}$ is strictly expanding. We also contrive to fit together the vector fields on each $T_{i}$ to yield a (partial) semiflow $\phi_{t}$ on $T=\bigcup_{i} T_{i}$. See Figure 2.2.

DEFINITION 2.15. A branched surface $T$ with semiflow $\phi_{t}$ constructed as above is called an abstract template. The $\alpha_{i}$ are called branch lines.

Consider an $f: U \rightarrow V$ which is hyperbolic with respect to a handle set $H=$ $\bigcup_{i} h_{i}$. We modify $H$ and $f$ so that for any $h_{i}, f\left(h_{i}\right) \cap h_{j}$ is connected $\forall_{j}$; using this method Figure 2.3a becomes Figure 2.3b. This creates more 1-handles in $H$ 


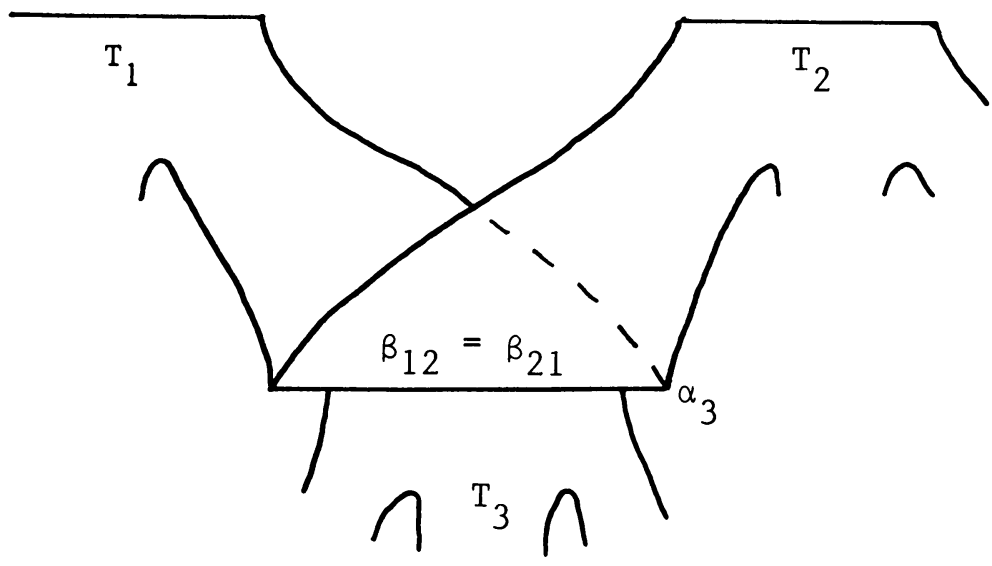

FIGURE 2.2

i

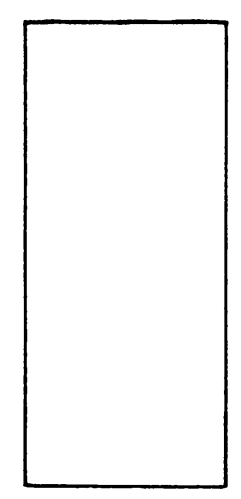

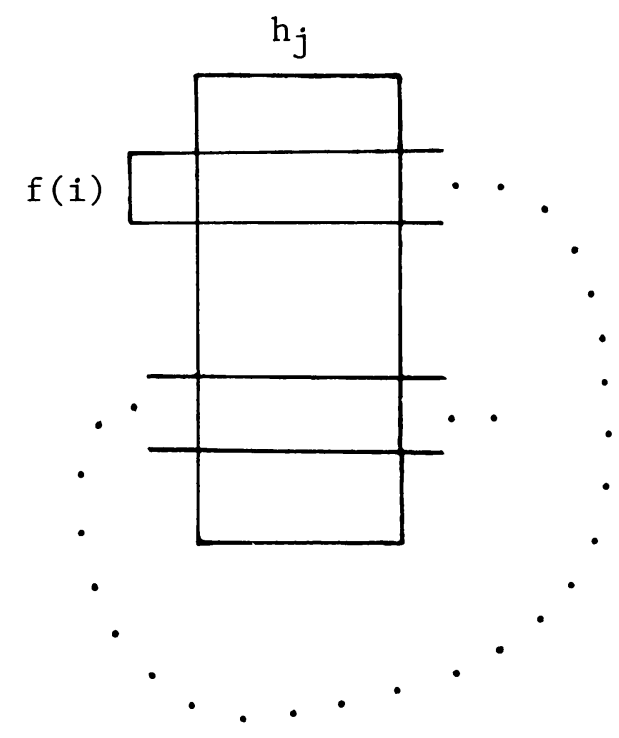

FIGURE 2.3a

but leaves the dynamics unchanged, for we have discarded only wandering points. Suppose we now have $n$ handles.

Next we assign some orientation data to each 1-handle: left-to-right for the horizontal direction and top-to-bottom for the vertical direction. We consider the horizontal direction as unstable, and the vertical as stable; choosing the unstable direction as primary, this yields a 2-dimensional orientation for each $h_{i}$.

Then given $h_{i}, f\left(h_{i}\right)$ hits $k_{i}$ handles, and we count these intersections from $f$ (left end of $h_{i}$ ) to $f$ (right end of $h_{i}$ ) using the assigned orientations. We say that the numbers $1, \ldots, k_{i}$ are horizontal crossing numbers of $h_{i}$. Similarly, given $h_{j}, m_{j}$ handles hit $h_{j}$ under $f$, and we count these intersections from the top of $h_{j}$ to the bottom, again using assigned orientations. We call the numbers $1, \ldots, m_{j}$ vertical crossing numbers of $h_{j}$. 

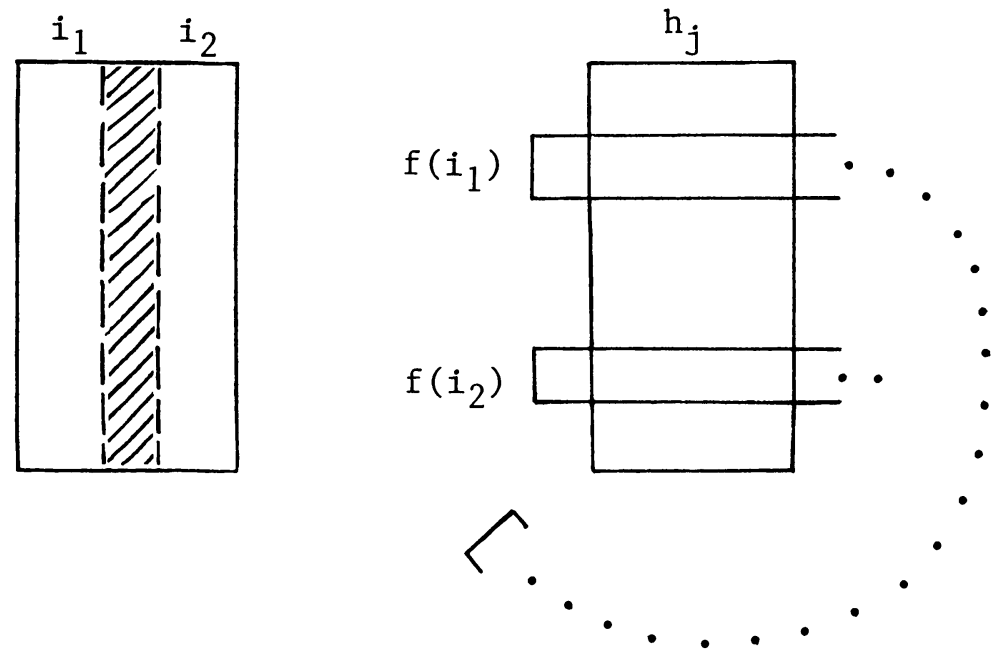

FIGURE 2.3b

We now create an $n \times n$ array $\left(a_{i j}\right)$ whose entries are either 0 or ordered triples: $a_{i j}=0$ if $f\left(h_{j}\right) \cap h_{i}=\varnothing$; the first entry in $a_{i j}$ is 1 (respectively -1 ) if $f\left(h_{j}\right) \cap h_{i} \neq$ $\varnothing$, with the unstable direction preserved (respectively reversed) by $f$; if $a_{i j} \neq 0$, its second entry is the horizontal crossing number of $h_{j}$ for $f\left(h_{j}\right) \cap h_{i}$; if $a_{i j} \neq 0$, its third entry is the vertical crossing number of $h_{i}$ for $f\left(h_{j}\right) \cap h_{i}$.

DEFINITION 2.16. An array $A$ constructed as above is called an order array for $H$ and $f$.

Lastly we introduce a matrix representation of a branched 1-manifold. We assume that an orientation has been assigned to each branch point--this would certainly be the case if the train track were embedded in an orientable surface. We then have locally, Figure 2.4.

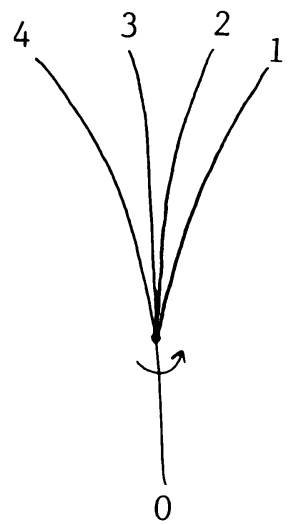

FIGURE 2.4

Now we assume that the train track has $n$ branch points. We will create an $n \times n$ matrix $B$ with each entry $b_{i j}$ consisting of a finite collection of symbols representing paths between branch points: $b_{i j}$ will contain $\left(\begin{array}{c}h \\ m\end{array}\right)$ if there is a path from branch pt. $\sharp i$ (branch $h)$ to branch pt. $\sharp j$ (branch $m$ ). 


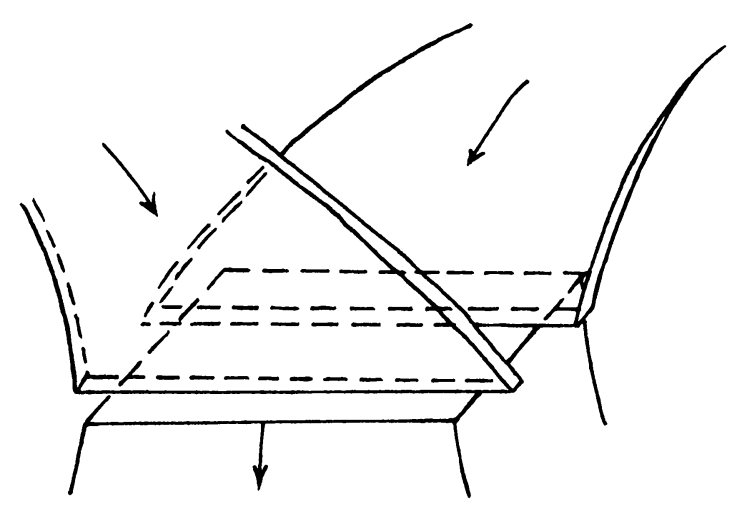

FIGURE 3.1

Note that $B$ is skew-symmetric, in a sense:

If

$$
b_{i j}=\left(\begin{array}{c}
h \\
m
\end{array}\right),\left(\begin{array}{l}
l \\
p
\end{array}\right), \ldots,\left(\begin{array}{l}
k \\
q
\end{array}\right)
$$

then

$$
b_{j i}=\left(\begin{array}{c}
m \\
h
\end{array}\right),\left(\begin{array}{l}
p \\
l
\end{array}\right), \ldots,\left(\begin{array}{l}
q \\
k
\end{array}\right) .
$$

For diagonal entries (paths from a branch point to itself), we choose the greater of the two branch numbers for the "superscript."

DEFINITION 2.17. The matrix $B$ in the above discussion is called a branched 1-manifold matrix.

3.

A. Branched 1-manifolds of handle sets. We now begin to describe the relationship involving templates, branched 1-manifolds, and laminations mentioned in the Introduction. Firstly we discuss a connection between abstract templates and handle sets.

LEMMA 3.1. There is a 1-1 correspondence between the set of handle sets and the set of abstract templates.

PROOF. Given an abstract template $T$ we form its associated thickened template $T^{\prime}$ by thickening $T$ in a transverse direction (in $S^{3}$ ). We do this in such a way that the (partial) semiflow on $T$ becomes a (partial) flow with hyperbolic structure of index 1 on $T^{\prime}$ : Branch lines become branch rectangles, and incoming sheets meeting at a branch line become disjoint incoming thickened sheets joined to a branch rectangle. See Figure 3.1. The flow on $T^{\prime}$ contracts in the new (formerly transverse) direction.

The associated handle set consists of the branch rectangles; i.e., the handle set is a cross section to the partial flow on $T^{\prime}$, and $f$ is the first-return map.

Conversely, suppose we have a handle set $H$. Then there is an $f: H \rightarrow f(H) \subset$ $V$, a compact neighborhood of $H$. We extend this to $\bar{f}: V \rightarrow V$ and form its suspension flow $\phi_{t}$. Restricting the domain of $\phi_{t}$ to $H$ and flowing forward gives us a thickened template, and collapsing this in the stable (contracting) direction yields a template. Q.E.D. 


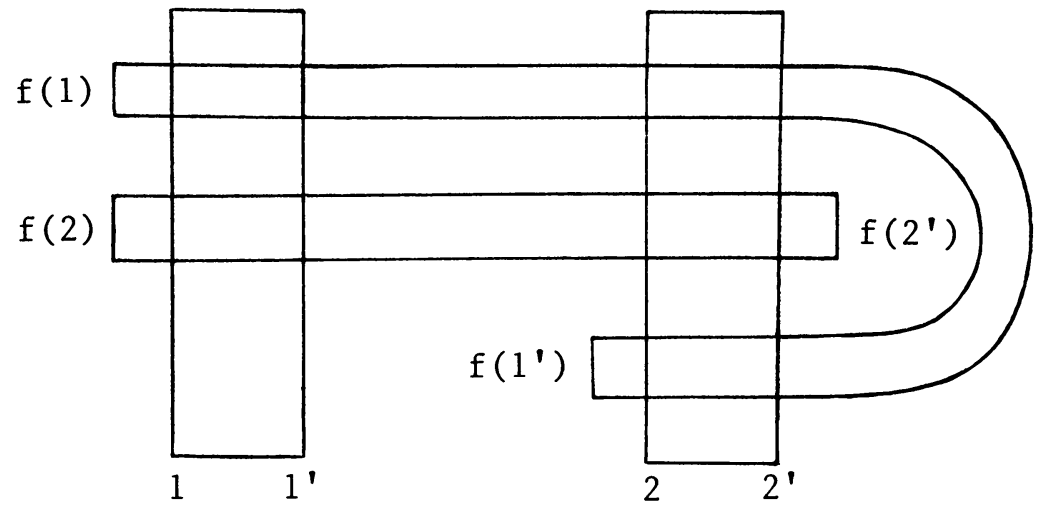

FIGURE 3.2

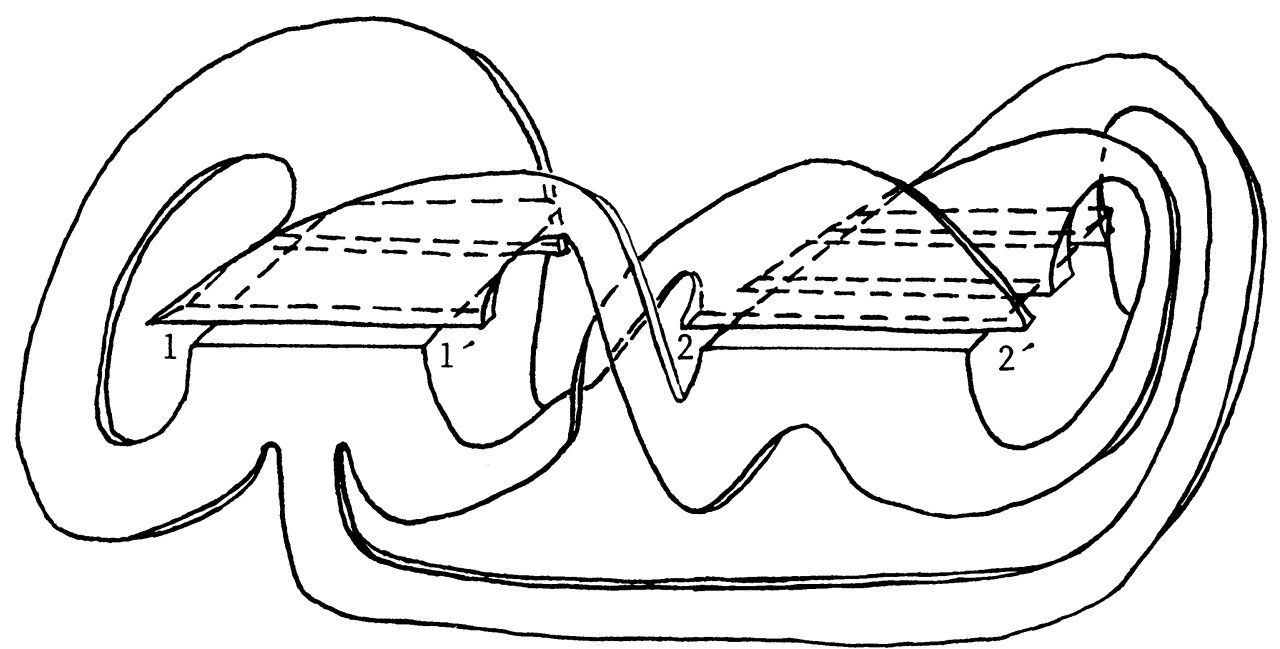

FIGURE 3.3

For example, consider the handle set in Figure 3.2. This becomes the thickened template of Figure 3.3.

There are now, because of Lemma 3.1, two equivalent ways to obtain an ordered branched 1-manifold from a handle set:

(1) as the boundary of the associated template, and

(2) from the train track neighborhood given in Proposition 2.14: collapsing each transverse leaf of this neighborhood to a point yields a branched 1-manifold.

PROPOSITION 3.2. Let $A$ be an order array representing a basic set $\Lambda$ in a Smale flow on an oriented 3-manifold $M$ with Lyapunov graph $\Gamma$. Then $A$ determines two ordered branched 1-manifolds, one labeled "backward," the other "forward."

PrOOF. $A$ is the label of a vertex $x$ in $\Gamma$; we restrict our attention to a connected neighborhood of $x$ which contains no other vertex. The branched 1-manifold $L_{f}$ derived from $A$ is then embedded in an oriented (because $M$ is) surface $S$ which is represented by a point(s) on an edge(s) just "below" $x$ in $\Gamma$. Thus, $L_{f}$ is an ordered branched 1-manifold, and is called the "forward" ordered branched 1-manifold. By 
considering the reverse flow, $A$ yields an "inverse" order array $A^{\prime}$, and the above reasoning gives $L_{b}$, the "backward" ordered branched 1-manifold. Q.E.D.

Notes. (1) The surfaces and branched 1-manifolds mentioned above need not be connected.

(2) The author has developed an algorithm for deriving a branched 1-manifold matrix for $L_{f}$ directly from $A$; likewise for $L_{b}$ and $A^{\prime}$. It is cumbersome, but a computer program could be written for it. See Theorem 3.2 of [3].

(3) In a branched 1-manifold matrix obtained from an order array, there are no occurrences of $\left(\begin{array}{l}0 \\ 0\end{array}\right)$. This follows from the stipulation in Definition 2.15 that each $\beta_{i j}$ be attached to an $\alpha_{k}$.

We have just established a way to derive a branched 1-manifold from an order array. To proceed in the opposite direction, we start with an abstract ordered branched 1-manifold $K$ with $n$ (even) branch points and try to build a handle set from this. We must group the $k$-pronged branch points in pairs for each $k$ :

DEFINITION 3.3. An abstract ordered branched 1-manifold along with information which assigns to each $k$-pronged branch point $x$ a partner $x^{\prime}$, another $k$-pronged branch point, $\forall k$ is said to be an abstract ordered branched 1-manifold with pairing information (data). We require that $\left(a^{\prime}\right)^{\prime}=a$.

PROPOSITION 3.4. An abstract ordered branched 1-manifold $L$ with pairing information determines at most one flow equivalence class of associated handle sets; without pairing data, it determines a finite number of such classes.

PROOF. Given a branched 1-manifold $L$ with $n$ (even) branch points and pairing data, we associate two paired branch points $k$ and $k^{\prime}$ with the ends of a handle. Then $n / 2$ handles have been created, and the number of vertical crossings for each is equal to the number of branches at the corresponding paired branch points; we number these as in Definition 2.16.

To define a potential $f$-potential in that some inconsistencies may arise that prevent the construction from satisfying certain properties - on this handle set, we shall attempt to draw it in the plane, connecting the crossings with untwisted open strips $\left(D^{1} \times \stackrel{\circ}{D}^{1}\right)$. We use the branched 1-manifold: if there is an edge from branch point $k$ to branch point $l$, we connect end $k$ to end $l$, using the cyclic order at the branch points to determine which vertical crossings to connect. This drawing may include undercrossings, but we are only concerned with the abstract handle set, not its immersion in $\mathbf{R}^{2}$. Let $B$ be the matrix for $L$, and denote vertical crossing numbers by "crossing $\sharp$." If

(a)

$$
\left(\begin{array}{l}
h \\
l
\end{array}\right) \in b_{i j}
$$

connect end $i$, crossing $\sharp h$, to end $j$, crossing $\sharp l$;

(b)

$$
\left(\begin{array}{c}
h \\
m_{j}-l+1
\end{array}\right) \in b_{i j^{\prime}},
$$

connect end $i$, crossing $\sharp h$, to end $j^{\prime}$, crossing $\sharp l$;

(c)

$$
\left(\begin{array}{c}
m_{i}-h+1 \\
l
\end{array}\right) \in b_{i^{\prime} j},
$$

connect end $i^{\prime}$, crossing $\sharp h$, to end $j$, crossing $\sharp l$; 
(d)

$$
\left(\begin{array}{c}
m_{i}-h+1 \\
m_{j}-l+1
\end{array}\right) \in b_{i^{\prime} j^{\prime}}
$$

connect end $i^{\prime}$, crossing $\sharp h$, to end $j^{\prime}$, crossing $\sharp l$;

(e)

$$
\left(\begin{array}{l}
h \\
0
\end{array}\right) \in b_{i j}
$$

draw $f$ (end $j$ ) and connect it to end $i$, crossing $\sharp h$; likewise for $j^{\prime}$ replacing $j$;

(f)

$$
\left(\begin{array}{c}
m_{i}-h+1 \\
0
\end{array}\right) \in b_{i^{\prime} j}
$$

draw $f$ (end $j$ ) and connect it to end $i^{\prime}$, crossing $\sharp h$; likewise for $j^{\prime}$ replacing $j$.

Note that it is immaterial which vertex/end in a pair is labeled "prime," for interchanging yields the same (potential) handle set, but with a different immersion in $\mathbf{R}^{2}$ in our drawing. More precisely, suppose we derive $H_{1}$ and $f_{1}$ with a certain labeling of $L$, and $H_{2}$ and $f_{2}$ arise from the same pairing data with some partners interchanged. Then $f_{2}=g \circ f_{1}$, where $g$ is a diffeomorphism, supported on planar neighborhoods of the handles involved in the interchange, which revolves each of these handles $180^{\circ}$ in the plane. $g$ does not affect the order array of the handle set(s). The above is true because the cyclic order of branches at paired branch points determines the 2-dimensional orientation of the associated 1-handle. Interchanging ends just yields different 1-dimensional orientations of leaves of the core and transverse foliations; steps (a)-(f) accommodate this.

For anyone with the patience to read through the above, an example may help. Given $L$ as in Figure 3.4, we form its matrix

$$
B=\begin{array}{cccc}
1 & 1^{\prime} & 2 & 2^{\prime} \\
1 \\
1^{\prime} \\
2 \\
2^{\prime}
\end{array}\left(\begin{array}{cccc}
\left(\begin{array}{c}
1 \\
0
\end{array}\right) & 0 & \left(\begin{array}{l}
2 \\
0
\end{array}\right) & 0 \\
0 & 0 & \left(\begin{array}{l}
1 \\
1
\end{array}\right),\left(\begin{array}{l}
2 \\
2
\end{array}\right) & \left(\begin{array}{c}
0 \\
1
\end{array}\right) \\
\left(\begin{array}{l}
0 \\
2
\end{array}\right) & \left(\begin{array}{l}
1 \\
1
\end{array}\right),\left(\begin{array}{l}
2 \\
2
\end{array}\right) & 0 & 0 \\
0 & \left(\begin{array}{l}
1 \\
0
\end{array}\right) & 0 & \left(\begin{array}{l}
2 \\
0
\end{array}\right)
\end{array}\right) .
$$

This yields Figure 3.5 .

As previously mentioned, the process yields only a potential handle set associated with $L$, for we may have created some inconsistencies with our added open strips: an annulus, which cannot be the homeomorphic image of a handle; incongruent matches, where a strip connects $f(i)$ to $f(j)$ with $j \neq i^{\prime}$; or a "reducible" handle set, which does not represent a single basic set. It is the only potential handle set associated with $L$, for the information contained in $L$ with pairing data forced our construction. The proposition's second statement follows from the fact that there are only a finite number of ways to pair the branch points of $L$. Q.E.D.

The pairing data is necessary to ensure a single associated flow equivalence class, as Figure 3.6 shows. Both $A$ and $B$ produce the branched 1-manifold of Figure 3.7. 


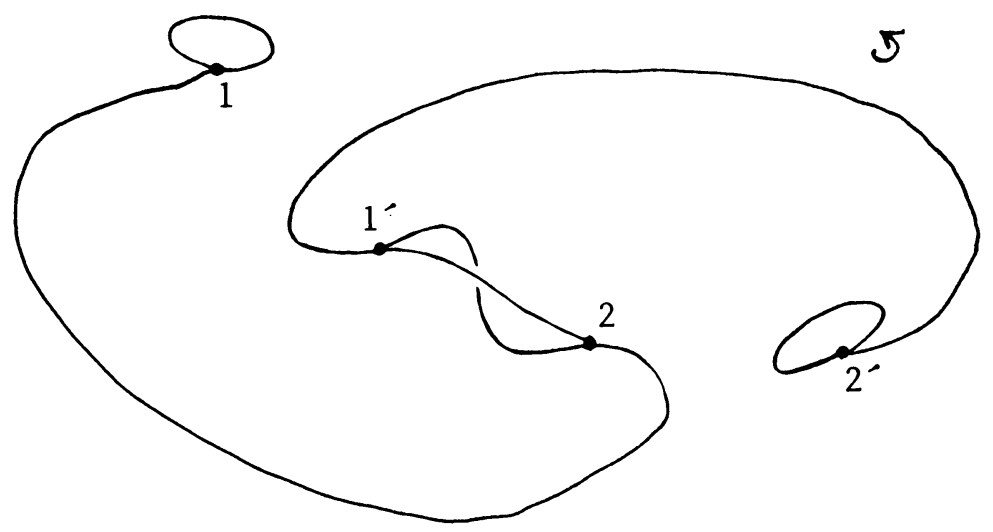

FIGURE 3.4

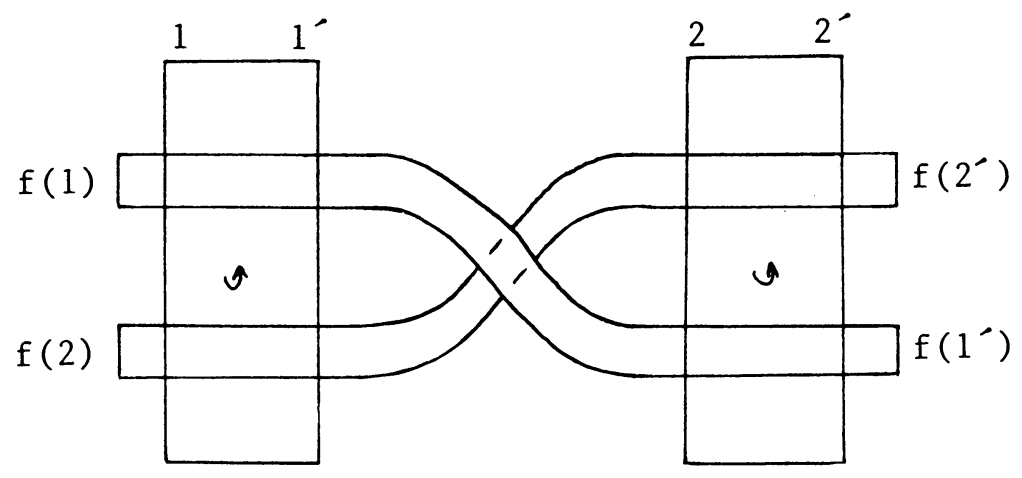

FIGURE 3.5

And yet, using Franks' result again (Theorem 2.10), $A$ is not flow equivalent to $B$ : Writing $A$ as a nonnegative matrix, we get

$$
A=\left(\begin{array}{lllll}
0 & 1 & 0 & 2 & 1 \\
0 & 1 & 0 & 2 & 0 \\
2 & 0 & 1 & 0 & 0 \\
0 & 1 & 1 & 0 & 1 \\
0 & 1 & 1 & 0 & 1
\end{array}\right)
$$

and we find that $\operatorname{Det}(I-A)=-4$. A similar calculation shows that $\operatorname{Det}(I-B)=4$.

We have just examined how one proceeds from branched 1-manifolds to handle sets, and what amount of additional information is needed to determine these handle sets to varying degrees. One particular piece of additional data which we have not yet analyzed is the "backward" branched 1-manifold: the branched 1-manifold derived from a handle set by considering the reverse flow and its first-return map. One might hope that we could build a unique handle set from a prescribed pair of branched 1-manifolds one labeled "forward," the other "reverse" - but Example 3.10 of [3] demonstrates that these two branched 1-manifolds, without pairing data, do not determine even the flow equivalence class of associated handle sets. 


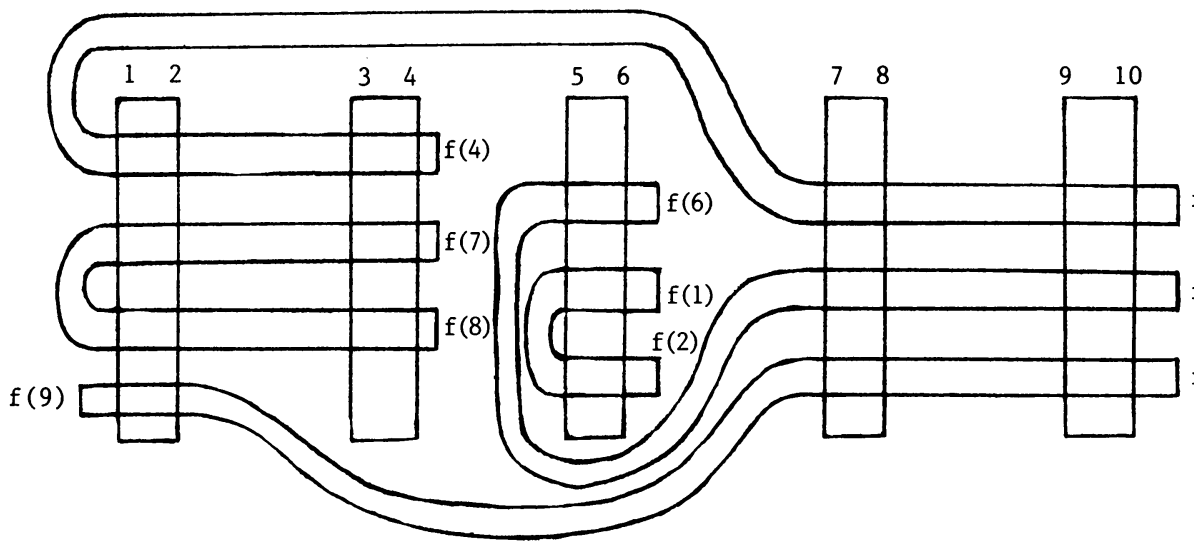

\section{$\underline{B}$}

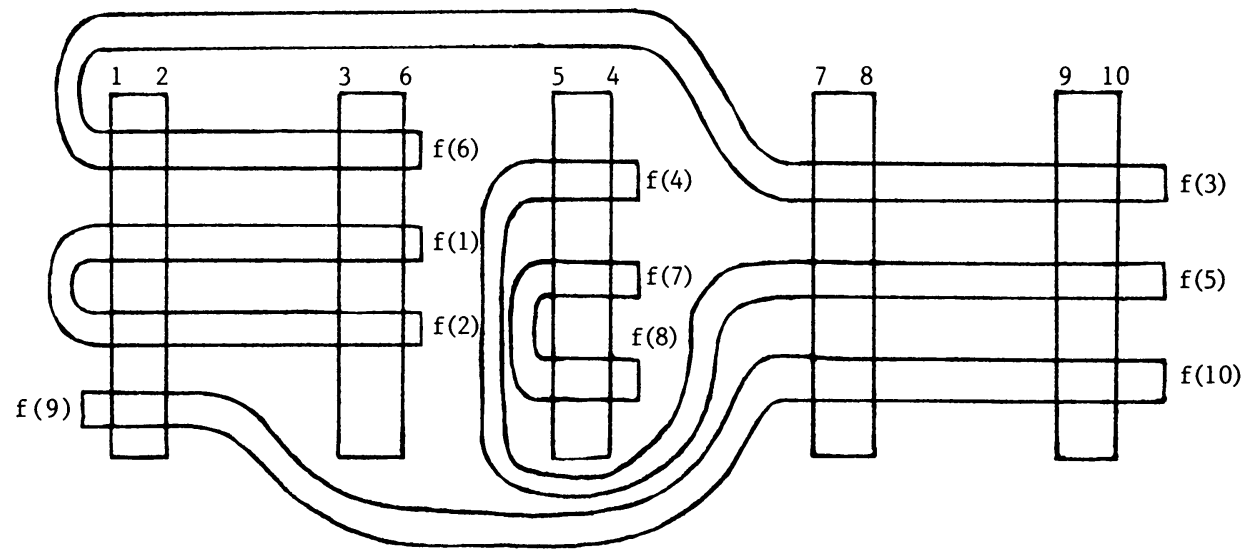

FIGURE 3.6

B. Laminations of handle sets. A major reason for studying branched 1-manifolds in our setting is their strong connection to $W^{u}(\Lambda)\left(W^{s}(\Lambda)\right.$ if we consider the backward branched 1-manifold), where $\Lambda$ is the basic set corresponding to our handle set $H=\bigcup_{i} h_{i}$.

LEMMA 3.6. Let $\Lambda^{\prime}$ be the suspension of $\Lambda=\bigcup_{n=-\infty}^{\infty} f^{n}(H)$, where $H$ is a handle set and $\Lambda^{\prime}$ is a hyperbolic basic set of $\phi_{t}$, a flow on an oriented 3-manifold $M$ with Lyapunov function $g$, where $\phi_{t} \mid H=$ suspension of $f$. Further suppose that $g\left(\Lambda^{\prime}\right)=c$ and $S=a$ component of $g^{-1}(c-\varepsilon)$ for $\varepsilon$ sufficiently small (in the same component of $g^{-1}([c-\varepsilon, c+\varepsilon])$ as $\left.\Lambda^{\prime}\right)$. If the order array $A$ describes $H$, and $K$ is the forward branched 1-manifold derived from $A, K$ carries $W^{u}\left(\Lambda^{\prime}\right) \cap S$. An analogous result holds for $W^{s}\left(\Lambda^{\prime}\right)$. 


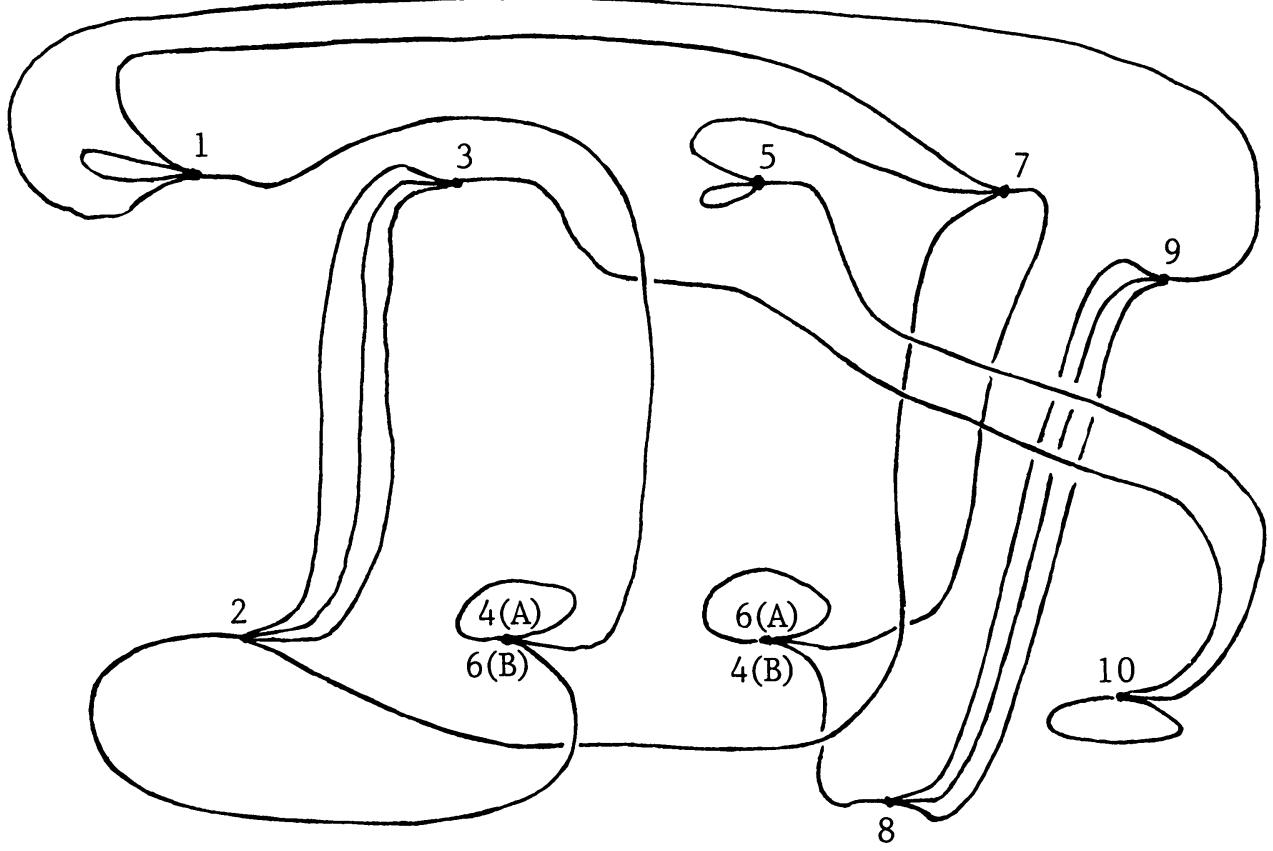

FIGURE 3.7
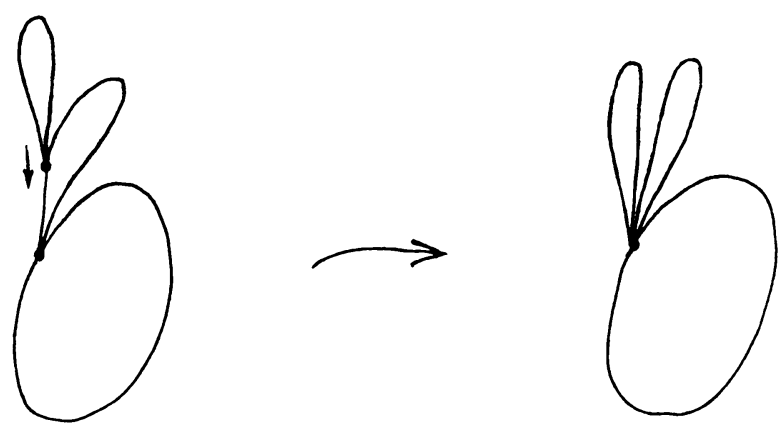

FIGURE 3.8

PROOF. Propositon 2.14 gives us a train track neighborhood $N \subset S$, and we note that $W^{u}\left(\Lambda^{\prime}\right) \cap S \subset N$. For each rectangle $k_{i}$ in $N$, we foliate $L_{i}=\bigcup_{x \in \Lambda} W_{i}^{u}(x) \subset k_{i}$; $L_{i} \equiv C_{i} \times I$, where $C_{i}$ is a Cantor set and $I=[-1,1]$. Then $W^{u}\left(\Lambda^{\prime}\right) \cap S=\bigcup L_{i}$. The fact that our branched 1-manifold $K$ carries this lamination follows by noting that $K$ is obtained by collapsing each transverse leaf of $N$ to a point. Q.E.D.

We notice that there is a way to alter a branched 1-manifold $K$ without changing the lamination carried by $K:$ a "combing" process discussed in [8]. It is pictured in Figure 3.8. The associated laminations are identical; we have just discarded portions of the train-track/lamination neighborhood.

We shall make this idea more precise: Start with a branch point $x$ and proceed away from $x$ along its 0 -branch to another branch point $y$ ( $y$ may equal $x$ ), then proceed in a similar manner from $y$ 's 0 -branch to branch point $z$; continue in this manner until we return to a branch point we have already visited; say, branch point 
$w$. If $w=x$, we do nothing for the moment. If $w \neq x$, we comb: All (nonzero) branches connected to $x$ are attached to $y$, respecting order, and $x$ (and the path from it to $y$ ) are eliminated. We renumber all the branches at $y$ if necessary. This process can be reflected in the branched 1-manifold matrix (see [3]).

Note that the above process does not allow for $\left(\begin{array}{l}0 \\ 0\end{array}\right)$ paths, but we have none in this context.

DEFINITION 3.7. The above manipulation of a branched 1-manifold $K$ is called combing $K$.

We continue combing, dealing with every branch point, until we acquire a finite number of circles along with 1-edged paths connecting them. We may encounter circles containing more than one branch point - there is no canonical way to comb these together. However, we see that the difference between two combing choices is described by a cyclic permutation of the paths connected to the circle, as shown in Figure 3.9. That is, the shift permutation $\sigma(x)=x+2(\bmod 3)$. Notice that this does not affect either the associated lamination or any paths connected to the inside of the above circle.
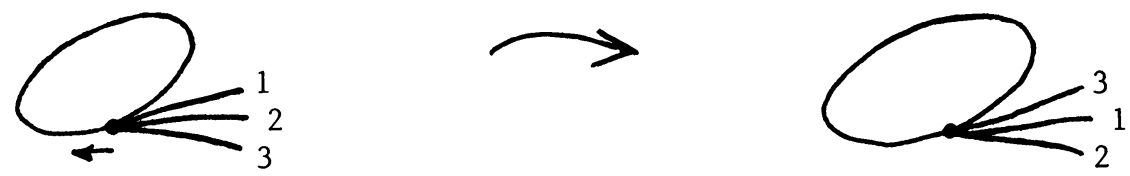

FIGURE 3.9

DEFINITION 3.8. Shift-combing along a circle in a branched 1-manifold means cyclically permuting all branches on a side (of the two sides) of the circle.

Consider a branched 1-manifold $K$ consisting of circles along with 1-edged paths connecting them, where each circle contains only one branch point. We say that $K$ is in combed form. Shift-combing induces an equivalence relation on the set of branched 1-manifolds in combed form: $L_{1} \sim L_{2}$ if $L_{1}$ can be shift-combed into $L_{2}$.

The equivalence classes of branched 1-manifolds can help us describe $W^{u}\left(\Lambda^{\prime}\right) \cap S$. Given a representative $L \in[L]$, the train track neighborhood $N$ associated to $L$ is made up of two types of 1-handles: one type is embedded in $S$, the other has just its interior embedded in $S$, with one end strictly contained in the other end. Then each nonembedded 1-handle $k_{i}$ yields a circle in $L_{i}=\bigcup_{x \in \Lambda} W_{i}^{u}(x) \subset k_{i}$ by a familiar argument involving a contracting map of an interval. Also notice that all other leaves of $L_{i}$ approach this circle asymptotically as we wind around $k_{i}$. The groups of leaves that do this correspond to the embedded 1-handles $k_{j}$ with at least one end contained in the longer end of $k_{i}$. This proves the following.

THEOREM 3.9 (THEOREM B). Let $\phi_{t}$ be a Smale flow on an orientable 3manifold $M$ with handle set $H=\bigcup h_{i}$ transverse to $\phi_{t}$ and $f$ the first-return map of $\phi_{t}$ restricted to $H$. Suppose $\Lambda^{\prime}=$ the suspension of $\Lambda=\bigcup_{n=-\infty}^{\infty} f^{n}(H)$ with corresponding order array $A$ and its derived branched 1-manifold $K$, and $g\left(\Lambda^{\prime}\right)=c$, a critical point of a Lyapunov function $g$ on $M$. Then for $S=$ a component of $g^{-1}(c-\varepsilon)$, in the same component of $g^{-1}([c-\varepsilon, c+\varepsilon])$ as $\Lambda^{\prime}, \varepsilon$ sufficiently small, $W^{u}\left(\Lambda^{\prime}\right) \cap S$ is a finite number of circles along with sets of the form $C \times \mathbf{R}, C$ a Cantor set, where $C \times t$ approaches a circle asymptotically as $t \rightarrow \infty$ and likewise 
for $t \rightarrow-\infty$; furthermore, $W^{u}\left(\Lambda^{\prime}\right) \cap S$ is carried by $K$. An analogous result holds for $W^{s}\left(\Lambda^{\prime}\right)$. Q.E.D.

The combing operation on branched 1-manifolds seems natural enough, and this feeling is solidified by the following: given a handle set $H=\bigcup h_{i}$ and $f$ hyperbolic with respect to $H$, let $A$ be the corresponding order array and $K$ its derived branched 1-manifold. If we then modify $K$ into $K^{\prime}$ by squeezing at a pair of branch points (see Figure 3.10) ( $K^{\prime}$ inherits pairing data from $A$ and $K$ ) we acquire a new order array $A^{\prime}$, with derived branched 1-manifold $K^{\prime}$. Let $C$ be the matrix given by $c_{i j}=$ first entry of $a_{i j}$ and $C^{\prime}$ be the matrix given by $c_{i j}^{\prime}=$ first entry of $a_{i j}^{\prime}$.
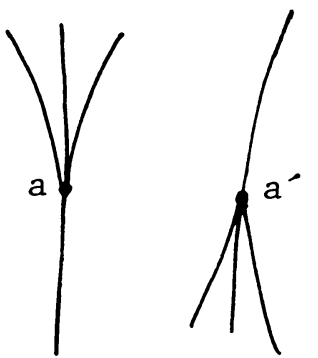
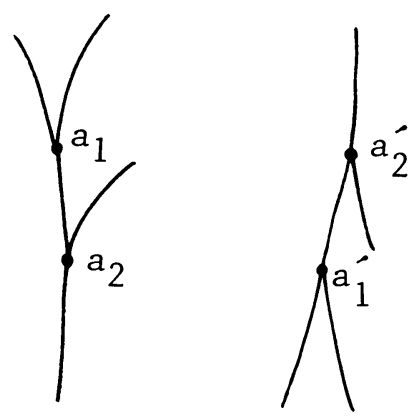

FIGURE 3.10

Proposition 3.10. $C$ and $C^{\prime}$ are flow equivalent.

ProOF. Assume we have squeezed at branch points $a$ and $a^{\prime}$, which correspond to branch rectangle $a_{0}$ in the associated thickened template $N$. Squeezing corresponds to encasing some of the incoming thickened sheets at $a_{0}$ in a box-like neighborhood $M$. However, $X=M-$ (thickened sheets) is wandering, because for each $x \in X$ there is a $T>0$ such that $\phi_{-t}(x) \notin N$ if $t>T$. Q.E.D.

We now analyze to what extent an equivalence class $[L]$ determines a 1-dimensional basic set. For an arbitrary $[L]$ to uniquely determine a flow equivalence class of associated handle sets, we know that we shall need to add to $[L]$ a type of pairing data, at least. However, this will be slightly different than before: here we label and pair, with "primes," the gaps between consecutive branches at each branch point of some $L \in[L]$ in the expectation, made feasible by Proposition 3.10, that we will squeeze each gap into a 3-pronged branch point. See Figure 3.11. By repeated squeezings, paired gaps become paired (3-pronged) branch points, and the rabel of each branch point is the label of the (only) gap associated to it. Note that this data for $L$ completely determines such data for any $L^{\prime} \in[L]$.

DEFINITION 3.11. An equivalence class $[L]$ with the data described above is said to be an equivalence class with pairing data.

This is not quite enough; the following shows that we need more information to determine a single flow equivalence class:

$$
A=\left(\begin{array}{ccc}
0 & 1,2,1 & 1,2,2 \\
1,1,2 & 1,3,1 & 0 \\
0 & 1,1,2 & 1,1,1
\end{array}\right)
$$




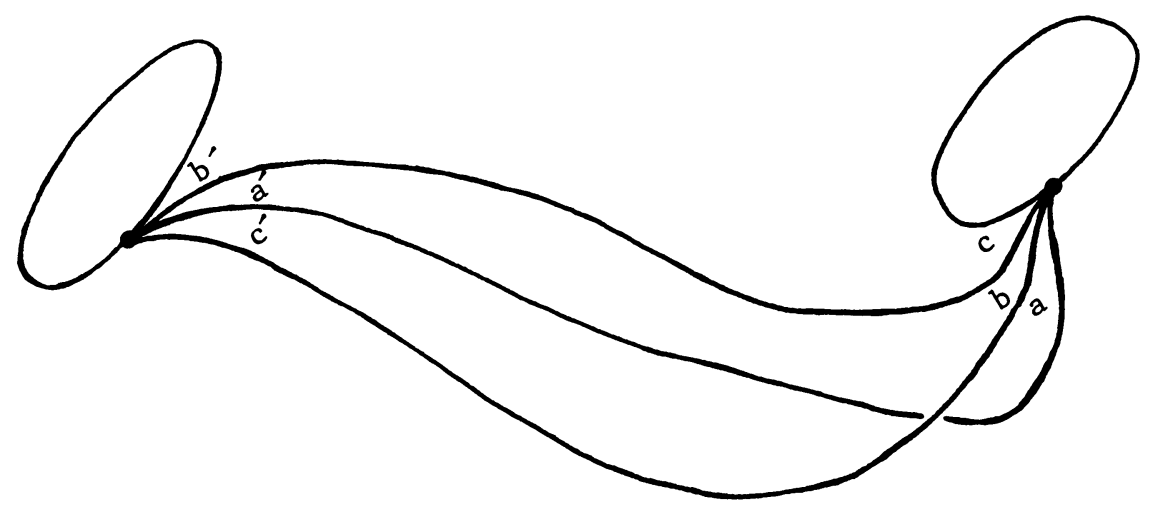

FIGURE 3.11

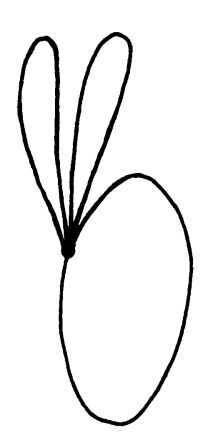

K

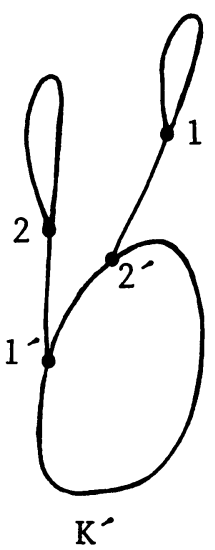

FIGURE 3.12
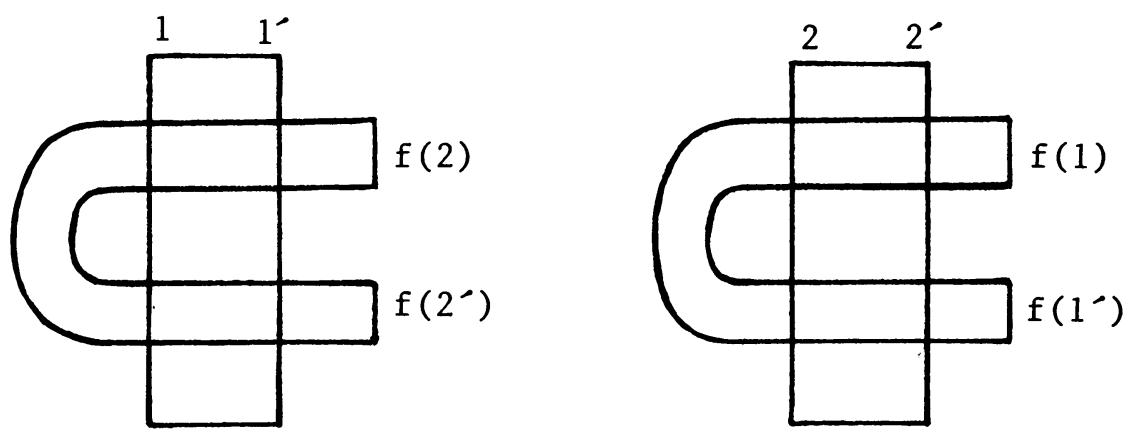

FIGURE 3.13

$$
B=\left(\begin{array}{ccc}
0 & 1,1,1 & 1,2,2 \\
1,2,2 & 1,2,1 & 0 \\
1,1,2 & 0 & 1,1,1
\end{array}\right)
$$

Both yield Figure 3.11. 
Let $A^{\prime}$ be the matrix given by $a_{i j}^{\prime}=$ first entry of $a_{i j}$ and $B^{\prime}$ be the matrix given by $b_{i j}^{\prime}=$ first entry of $b_{i j}$. Then $\left|I-A^{\prime}\right|=-1 \neq 0=\left|I-B^{\prime}\right|$, so by Theorem 2.10, $A$ and $B$ are not flow equivalent.

THEOREM 3.12 (THEOREM C). An equivalence class $[L]$ with pairing data and squeezing data (numbering each pair of gaps, telling us in which order to squeeze), determines a unique flow equivalence class of the associated handle sets, possibly the empty class.

ProOF. We start with such an $L \in[L]$. If the number of branch points in $L$ is even and the pairing data on the gaps yields a well-defined pairing data on the branch points - for example, if one branch point has gaps labeled $a$ and $b$, and another has gaps labeled $a^{\prime}$ and $b^{\prime}$, we can pair these two branch points-we use Proposition 3.4 to find an associated handle set; if we find one, Proposition 3.10 implies that any squeezing of $L$ in pairs will produce a flow-equivalent handle set. If we do not find a handle set from Proposition 3.4 we squeeze $L$, using the squeezing data to tell us which pair to squeeze, into $L^{\prime}$ and proceed through the above argument with this. Q.E.D.

It should be noted that by squeezing an unrealizable branched 1-manifold $K$, we can create a realizable $K^{\prime}$. See Figure 3.12. Now $K$, having an odd number of branch points, cannot be realized as the boundary of a template. However, $K^{\prime}$ is derived from Figure 3.13.

4. We are now ready to address some embedding issues: ordered branched 1manifolds in surfaces, and thickened templates in flows on 3-manifolds.

We start with an abstract template corresponding to an order array $A$. To acquire an associated Smale flow on an oriented 3-manifold, we must, among other things, embed the template's boundary, an ordered branched 1-manifold $K$, in an oriented surface.

DEFINITION 4.1. The genus of $K$ is the least genus of all oriented surfaces in which $K$ can be embedded.
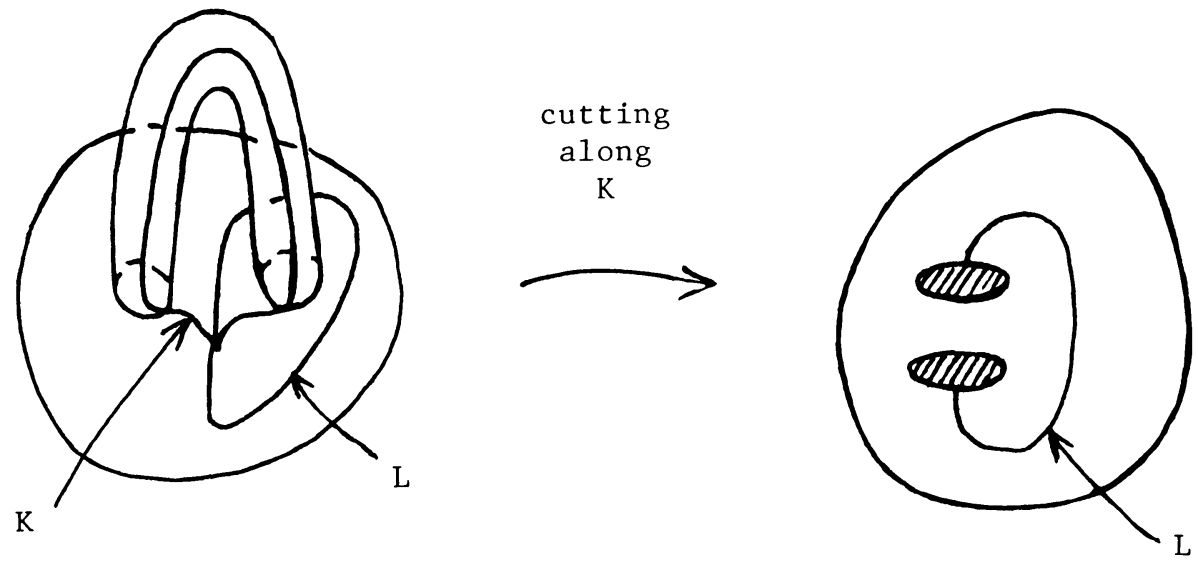

FIGURE 4.1 


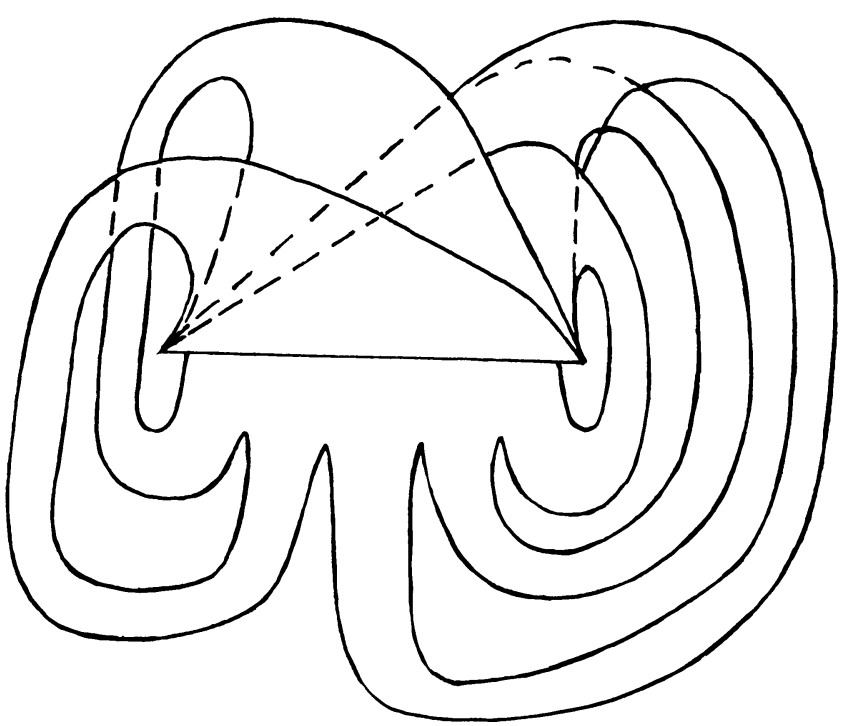

FIGURE 4.2

Note that for any $K$ and $K^{\prime}$ such that [combed form of $K$ ] $=$ [combed form of $K^{\prime}$ ], genus of $K=$ genus of $K^{\prime}$, for combing can be done with an arbitrarily small $C^{1}$-perturbation.

THEOREM 4.2. Suppose that $\phi_{t}$ is a flow on an orientable 3-manifold $M$ with Lyapunov function $g$ and $\Lambda^{\prime}$ is a 1-dimensional hyperbolic basic set of $\phi_{t}$, so that $\Lambda^{\prime}$ is the suspension of $\Lambda=\bigcap_{n=-\infty}^{\infty} f^{n}(H)$, where $H$ is a handle set and $\phi_{t} \mid H=$ suspension of $f$. Let $g\left(\Lambda^{\prime}\right)=c$ and pick $\varepsilon>0$ sufficiently small so that there is no other basic set in the component of $g^{-1}([c-\varepsilon, c+\varepsilon])$ which contains $\Lambda^{\prime} ;$ call this component $X$, and let $S_{1}, \ldots, S_{m}$ be the components of $g^{-1}(c-\varepsilon)$ in $X$. If $A$ is the order array associated to $\Lambda$ and $K$ is its derived branched 1-manifold, which decomposes into components $K_{1}, \ldots, K_{j}$, then $g\left(S_{i}\right) \geq \sum_{t} g\left(K_{t}\right)$ with $t$ ranging through all values such that $K_{t}$ is embedded in $S_{i}$, where $g(\cdot)$ denotes "genus of." Furthermore, $m \leq j$.

PROOF. $m$ cannot exceed $j$, for each component $S_{i}$ must intersect $W^{u}\left(\Lambda^{\prime}\right)$, and each $K_{h}$, being connected, must lie in a single $S_{i}$.

As for the first statement, pick an arbitrary $S_{i}$. Up to homeomorphism, we can consider $S_{i}$ to be $S^{2}$ with $g\left(S_{i}\right)$ handles attached in an untwisted, unlinked way. Suppose $K_{t_{1}}, \ldots, K_{t_{t}}$ are the components of $K$ in $S_{i}$. By cutting along $K_{t_{1}}$ as in Figure 4.1 at the $g\left(K_{t_{1}}\right)$ handles of $S_{i}$ which intersect $K_{t_{1}}$, we turn $S_{i}$ into $S_{i}^{1}$, a surface with boundary where $g\left(S_{i}^{1}\right) \leq g\left(S_{i}\right)-g\left(K_{t_{1}}\right)$.

Because the $K_{j}$ are disjoint, $K_{t_{2}}, \ldots, K_{t_{l}}$ are embedded in $S_{i}^{1}$. Repeating this process for each $K_{t_{j}}, 2 \leq j \leq l$, yields $S_{i}^{l}$ where $0 \leq g\left(S_{i}^{l}\right) \leq g\left(S_{i}\right)-\sum_{j=1}^{l} g\left(K_{t_{j}}\right)$. The desired inequality follows. Q.E.D.

We say that an order array $A$ can be realized in a flow $\phi_{t}$ if $A$ corresponds to a basic set of $\phi_{t}$ as in the hypotheses of the preceding theorem. When a branched 1-manifold $K$ decomposes into $K_{1}, \ldots, K_{j}$, we call $\max _{1 \leq i \leq j}$ \{genus of $K_{i}$ \} the genus of $K$. 
THEOREM 4.3 (THEOREM A). Let $A$ be an order array with derived branched 1-manifold $K$, and suppose that $\phi_{t}$ is a nonsingular Smale flow on a compact oriented 3-manifold $M$, with $H_{1}(M ; Q)=0$. A necessary condition for $A$ to be realized in $\phi_{t}$ is that the genus of $K \leq 1$.

PROOF. Lemma 2.2 of [6] implies that the surfaces $S$ of Theorem 4.2 are each tori: the Poincaré-Hopf formula ensures this, because the $S_{i}$ are transverse to a nonsingular flow. Our result then follows from Theorem 4.2. Q.E.D.

Theorem 4.3 is a simple extension of Theorem 2 in [4] to the set of order arrays. Consider the template $T$ in Figure 4.2. Let $K=\partial T$. $T$ corresponds to the structure matrix $A=[\mathbf{5}]$, so $Z /(I-A) Z \cong Z_{4}$ is cyclic; therefore there is no obstruction to its embedding in a nonsingular Smale flow on $S^{3}$ from the earlier theorem.

Modifying as in Figure 2.3, we describe $T$ by the order array

$$
A^{\prime}=\left(\begin{array}{lllll}
1,1,4 & 1,1,5 & 1,1,2 & 1,1,1 & 1,1,3 \\
1,2,4 & 1,2,5 & 1,2,2 & 1,2,1 & 1,2,3 \\
1,3,4 & 1,3,5 & 1,3,2 & 1,3,1 & 1,3,3 \\
1,4,4 & 1,4,5 & 1,4,2 & 1,4,1 & 1,4,3 \\
1,5,4 & 1,5,5 & 1,5,2 & 1,5,1 & 1,5,3
\end{array}\right)
$$

which has derived branched 1-manifold $K^{\prime}$; note that genus of $K^{\prime}=$ genus of $K$. The matrix of $K$ is

$$
\left(\begin{array}{cc}
\left(\begin{array}{l}
2 \\
0
\end{array}\right) & \left(\begin{array}{l}
1 \\
4
\end{array}\right),\left(\begin{array}{l}
3 \\
1
\end{array}\right),\left(\begin{array}{l}
4 \\
5
\end{array}\right),\left(\begin{array}{l}
5 \\
2
\end{array}\right) \\
\left(\begin{array}{l}
4 \\
1
\end{array}\right),\left(\begin{array}{l}
1 \\
3
\end{array}\right),\left(\begin{array}{l}
5 \\
4
\end{array}\right),\left(\begin{array}{l}
2 \\
5
\end{array}\right) & \left(\begin{array}{l}
3 \\
0
\end{array}\right)
\end{array}\right)
$$

which has genus 2. Therefore $A^{\prime}$ cannot be realized in a nonsingular Smale flow on $S^{3}$.

\section{REFERENCES}

1. R. Bowen, One-dimensional hyperbolic sets for flows, J. Differential Equations 12 (1972), 173179.

2. C. Conley, Isolated invariant sets and the Morse index, CBMS Regional Conf. Ser. in Math., no. 38, Amer. Math. Soc., Providence, R. I., 1978.

3. G. Frank, Templates, branched one-manifolds, and laminations, Ph.D. Thesis, Northwestern Univ., 1985.

4. J. Franks, Symbolic dynamics in flows on three-manifolds, Trans. Amer. Math. Soc. 279 (1983), 231-236.

5. _ Flow equivalence of subshifts of finite type, Ergodic Theory and Dynamical Systems 4 (1984), 53-66.

6. Nonsingular Smale flows on $S^{3}$, I.H.E.S. preprint, 1983.

7. S. Smale, Differentiable dynamical systems, Bull. Amer. Math. Soc. 73 (1967), 744-817.

8. R. Williams, Classification of one-dimensional attractors, Proc. Sympos. Pure Math., vol. 14, Amer. Math. Soc., Providence, R. I., 1970, pp. 341-361.

9. __ Expanding attractors, Publ. Math. Inst Hautes Études Sci. 43 (1974), 161-203.

10. W. Wilson, Smoothing derivatives of functions and applications, Trans. Amer. Math. Soc. 139 (1969), 413-428.

Department of Mathematics and Statistics, University of South Alabama, Mobile, Alabama 36688 